PO39

\title{
COLOR MATCHING CONSIDERATION ON THE EFFECT OF IPRGC FOR COLOR REPRODUCTION ON DISPLAY DEVICE
}

\author{
Kota Akiba et al.
}

DOI 10.25039/x47.2020.PO39

\section{Paper accepted for the $5^{\text {th }}$ CIE Symposium on Colour and Visual Appearance}

The paper was selected by the International Scientific Committee (ISC) for presentation at the 5th CIE Symposium on Colour and Visual Appearance, Hong Kong, CN, April 21-22, 2020, which, due to the corona pandemic, could not take place. The paper has not been peer-reviewed by CIE.

\section{(C) CIE 2020}

All rights reserved. Unless otherwise specified, no part of this publication may be reproduced or utilized in any form or by any means, electronic or mechanical, including photocopying and microfilm, without permission in writing from CIE Central Bureau at the address below. Any mention of organizations or products does not imply endorsement by the CIE.

This paper is made available open access for individual use. However, in all other cases all rights are reserved unless explicit permission is sought from and given by the CIE.

CIE Central Bureau

Babenbergerstrasse 9

A-1010 Vienna

Austria

Tel.: +4317143187

e-mail: ciecb@cie.co.at

www.cie.co.at 


\title{
PO39 \\ COLOUR MATCHING CONSIDERATION ON THE EFFECT OF IPRGC FOR COLOUR REPRODUCTION ON DISPLAY DEVICE
}

\author{
Akiba, K. ${ }^{1}$, Tanaka, M. ${ }^{2}$, Horiuchi, T. ${ }^{1}$ \\ ${ }^{1}$ Graduate School of Science and Engineering, Chiba University, Chiba, JAPAN \\ ${ }^{2}$ College of Liberal Arts and Sciences, Chiba University, Chiba, JAPAN \\ midori@chiba-u.jp
}

\begin{abstract}
At the beginning of this century, intrinsically photoreceptive retinal ganglion cells (ipRGCs), new photoreceptors different from cones and rods, were discovered. The aim of this study was to experimentally verify the influence of ipRGCs on the colour reproduction of a display. In the experiment, we performed perceptual colour matching between colour patches and displays. Although colorimetric reproduction of the colour patch was used for the initial image of the display, the colour matching results were largely out of colorimetric colour reproduction. Since this result suggests that ipRGCs may affect colour reproduction of displays, a correction equation that accounts for the influence of ipRGCs was derived. Applying this correction formula improved the results. It is considered possible to accurately display the colour of a real object on a display using a correction equation that accounts for the effect of ipRGCs.
\end{abstract}

Keywords: ipRGC, colour reproduction, colour perception, display

\section{Introduction}

At the beginning of this century, cones and photoreceptors other than rods were discovered in the retina of mammals and were named "intrinsically photosensitive retinal ganglion cells" (ipRGCs) [1]. The ipRGC is a special ganglion cell containing the visual substance melanopsin. The spectral sensitivity function [2] shown in Figure 1 was defined by the Commission Internationale de I'Eclairage (CIE) in 2018 and had a peak at around $490 \mathrm{~nm}$. The ipRGC affects non-imaging functions, such as regulation of the circadian rhythm and pupillary light reflection, compared to imaging functions that form the image of an object, such as cones and rods [3]. However, recent studies have reported that ipRGC affects human visual perception through changes in the amount of stimulation for visual perception. For example, to verify the effect of ipRGC on visual perception, we used the silent-substitution method, which changes only the amount of stimulation to ipRGC without changing the amount of stimulation to the cone by manipulating the shape of the spectral distribution [4]. Considering a method for stimulating only melanopsin cells, colour stimuli were created using a light-emitting diode (LED) mounted on an integrating sphere as a light source, and experiments were performed. Figure 1 shows the sensitivity of the long, medium, short-wavelength (LMS) cone and the ipRGC overlap. All these reported experiments were performed in a tightly controlled environment.

In current displays, colour reproduction is performed colorimetrically based on the perception amount of the LMS cone in photopic vision. However, previous studies have suggested that ipRGC may affect visual perception. If ipRGCs influence colour perception, in addition to the amount perceived by the LMS cone, it is necessary to consider the effect of ipRGC on the colour reproduction of display devices. In our previous study [5], we attempted to analyze a fundamental colour matching experiment in which patches and display reproduction colours were visually juxtaposed. It indicated that ipRGCs might contribute to the colour vision pathway. However, since the stimuli were visually placed side by side, observing them with a single eye was necessary.

The aim of this study was to verify the effect of ipRGCs on the display in an experimental environment where patches and display reproduction colours were physically juxtaposed. Furthermore, when it was suggested that the colour reproduction of a display is different from 
that of a conventional display, we constructed a model for evaluating the colour reproduction accuracy of a new display constructed using the values obtained from the experimental results.

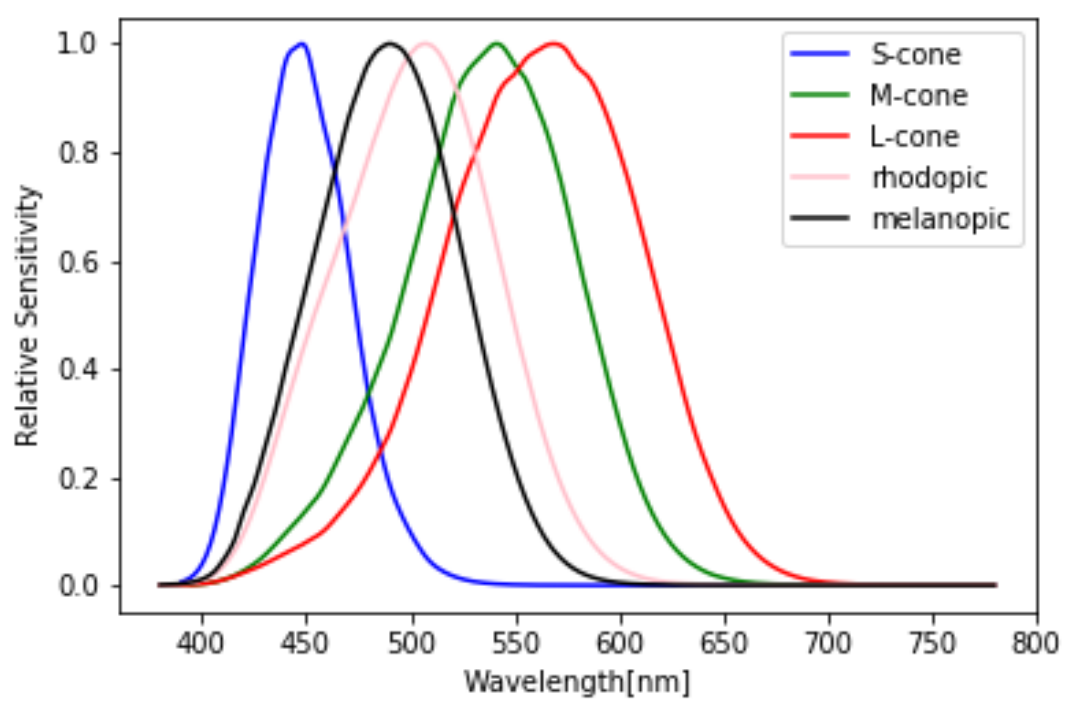

Figure 1 - Relative sensitivity of S-, M-, and L-cones and rhodopic and melanopic irradiance

\section{Experiments}

In the experiment, perceptual colour matching was performed between colour patches, and the colour patches were reproduced on a display under $6000 \mathrm{~K}$ by $4500 \mathrm{Ix}$ LED illumination. The spectral distributions of both colour stimuli were different. Therefore, matching results were expected without the smallest colour difference in the case that the ipRGCs influence colour perception. To control the influence of rods, we used a high-brightness liquid crystal display (SHARP PN-A601) after colour calibration. Drawing papers toned by Japan Colour Enterprise Co., Ltd. to the colour of the X-Rite ColorChecker were used as the real colour patches. Seven colours (red, blue, moderate red, blue sky, magenta, cyan, and white) with comparatively high reproducibility from 24 colour patches were used as the colour stimuli in this experiment. Many short- and long-wavelength components containing a colour were selected in addition to confirming the effect at approximately $490 \mathrm{~nm}$, which is the peak visibility of ipRGCs. The CIE1976 $\Delta \mathrm{E}$ colour difference was an average of 1.42 and a maximum of 3.15 (cyan).

In the experiment, colour matching was performed between a colour patch and a reproduced image on a high-brightness display. Since the spectral distributions of both colours are different, it is hypothesized that if ipRGC affects colour perception, the colour difference will not always be minimal. Figure 2 shows the experimental environment. The subjects were six observers (5 men and 1 woman) with normal colour vision. The experiment was performed twice to evaluate the stability of the results. The viewing distance from the observer to the display and colour patch was set at $150 \mathrm{~cm}$, and the experimental environment was designed in a way that both stimuli could be physically juxtaposed and observed with both eyes. The viewing angle of each colour stimulus was $3.4^{\circ}$. It was shielded from light by a black paper, except for the part showing the colour of the display. The colour matching procedure was performed by independently adjusting the hue, saturation, and lightness. The colorimetric reproduction colour with the smallest colour difference was displayed for each stimulus as the initial colour. The experiment was performed after the training process for the observers to become accustomed to toning. The adjusted colour on the display device was measured using a spectroradiometer (KONICA MINOLTA CS-2000) after the colour matching experiment. 


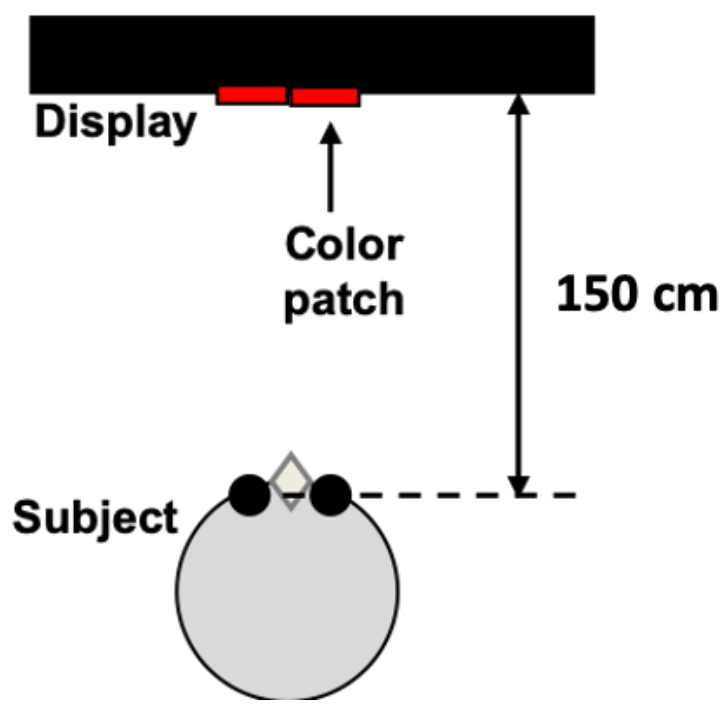

Figure 2 - Experimental environment

\section{Results and discussion}

The CIE 1976 Delta E colour difference between the reproduced colour and the colour patch was calculated after colour matching. The outliers for each colour of the experimental results were derived using the Smirnov-Grubbs test. Figure 3 shows the average statistical results of two experiments performed by six observers for each colour stimulus, together with the colour difference of colorimetric colour reproduction (initial colour). The deviation in the figure indicates the standard deviation. As shown in Figure 3, the colour difference average of the seven colours of colorimetric colour reproduction was 1.4 , and it changed to 3.9 after colour matching. In particular, the errors for the blue and white patches were large, and variations were observed among the observers.

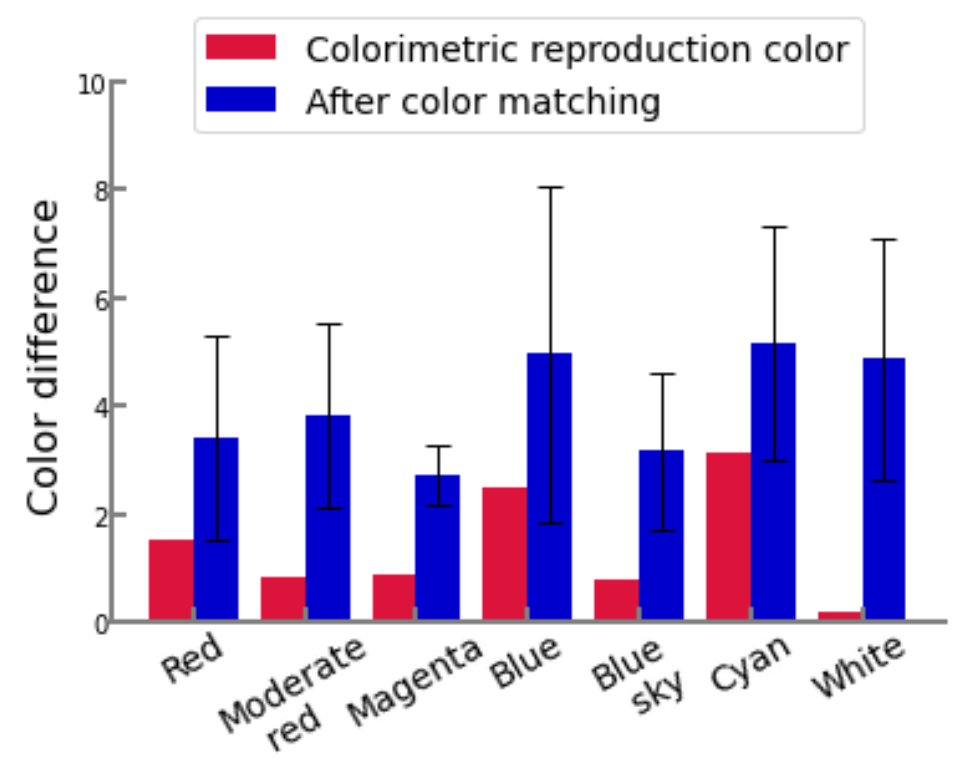

Figure 3 - Average colour difference among the six observers

The ratio of colour difference, i.e. $\Delta L^{*}: \Delta a^{*}: \Delta b^{*}$, was $0.26: 0.30: 0.44$. Focusing on the blue stimulus overlapping with the sensitivity of ipRGCs, the colour difference ratio was $0.12: 0.36: 0.52$, and the colour difference of $b^{*}$ was large. This result indicates that ipRGCs may also contribute to the colour vision pathway. 
The RGB colour-matching function of CIE1931 was derived from colour matching experiments using Guild and Light. Because this colour-matching function includes negative terms, the CIE1931 XYZ colour matching function was derived by basis transformation, which assumes that the LMS signals are independent. However, we hypothesized that the LMS signal would reach the ganglion cells and that the LMS signal would be biased by ipRGCs in the ganglion cells. Therefore, the correction formula of CIE XYZ obtained by correcting each value of CIE $X Y Z$ with the ipRGC absorption rate was derived by regression with the dependent variable as "CIE XYZ of a real colour patch" and the independent variables as "ipRGC absorption rate" and "CIE XYZ of the reproduced colour on the display after colour matching." Here, for the regression, 189 datasets obtained by removing the outliers from the XYZ data observed 12 times for the seven colour stimuli were used. The ipRGC absorption rate was calculated using the spectral sensitivity of ipRGC [2] and spectral distribution of the reproduced colours on the display. The modified $X Y Z$ values, $X Y Z_{i p R G C}$, are derived by the ipRGC effect as follows:

$$
X Y Z_{i p R G C}=-9.479+107.5 \times i p R G C+0.9277 \times X Y Z_{m}-(1)
$$

where $X Y Z_{m}$ represents the CIE XYZ values of the reproduced colour on the display device after the colour matching, including the impact of ipRGCs. The variable ipRGC is the ipRGC absorption rate of the reproduced colour. By adopting the modified XYZ values, Figure 4 presents the average colour difference among the six observers. According to the description in the figure, after applying the modified XYZ values, the CIE Delta E colour difference after colour matching worsened to an average of 5.6. The ratio of colour difference was 0.22:0.31:0.47.

Since the absorbance spectrum of ipRGCs has a peak on the short wavelength side, we modified only the Z-value of the display after colour matching. Figure 5 shows the average colour difference among the six observers. The colour difference using the correction formula improved to an average of 3.5 for the seven colours. In particular, improved results were obtained for the colour stimuli containing many short wavelength components. This suggested that the absorption component of ipRGCs affected the $z$-value in the tristimulus values.

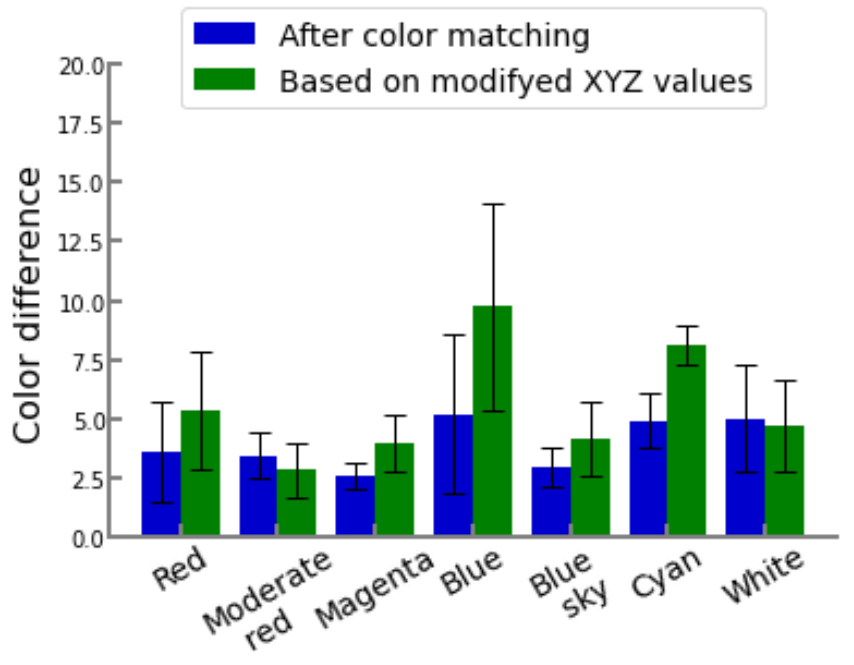

Figure 4 - Average colour difference among the six observers after applying the modified $\mathrm{XYZ}$ values 


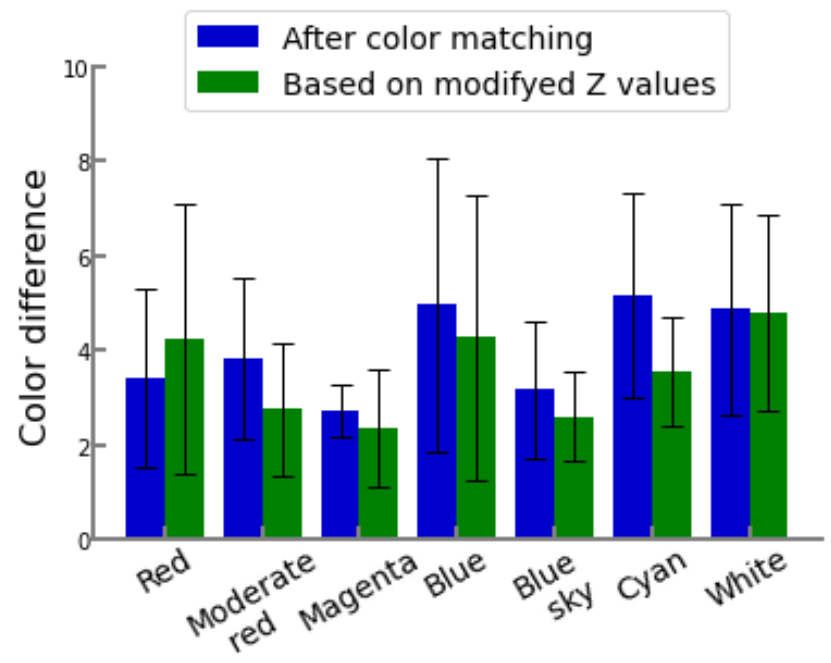

\section{Figure 5 - Average colour difference among the six observers after applying the modified Z} value

\section{Conclusions}

In the colour matching experiment using a high brightness display, colour perception of the display was influenced by ipRGCs. This effect could be confirmed even when colour matching was performed for the juxtaposed stimuli in the same way as in the previous study that included a single eye. We assumed that the LMS signal was biased by ipRGCs in the ganglion cells. The study also provided a derivation of the correction formula of CIE XYZ acquired by correcting $X Y Z$ values with the ipRGC absorption rate. In particular, by considering the CIE $Z$ value, the colour difference improved and suggested the necessity of ipRGCs in colour perception of displays.

Further analysis is required to derive a more appropriate correction formula while considering the effect of ipRGCs on the perceptual model. In general, it is expected that the sensitivity of ipRGCs and cone sensitivity depend on the observer; therefore, we need to consider an experimental design that takes this into consideration.

\section{Acknowledgment}

This work was supported by JSPS KAKENHI Grant Number 19 K12038.

\section{References}

1. D.M. Berson, F.A. Dunn, and M. Takao "Phototransduction by Retinal Ganglion Cells That Set the Circadian Clock" SCIENCE, vol 295, 8 February 2002

2. CIE System for Metrology of Optical Radiation for ipRGC-Influenced Responses to Light (2018)

3. S. Hattar, H.-W. Liao, M. Takao, D.M. Berson, and K.-W. Yau "Melanopsin-Containing Retinal Ganglion Cells: Architecture, Projections, and Intrinsic Photosensitivity" SCIENCE, vol 295, 8 February 2002

4. T.M. Brown, S. Tsujimura, A.E. Allen, J. Wynne, R. Bedford, G. Vickery, A. Vugler, and R.J. Lucas "Melanopsin-Based Brightness Discrimination in Mice and Humans" Current Biology, 22, pp.1134- 1141 (2012)

5. K. Akiba, M. Tanaka, and T. Horiuchi "Experimental consideration on the effect of ipRGC for color reproduction on display device" International Color Association, Buenos Aires, Argentina, October 2019 